\title{
Graph Embedding Using an Edge-Based Wave Kernel
}

\author{
Hewayda ElGhawalby ${ }^{1,2}$ and Edwin R. Hancock ${ }^{1, \star}$ \\ 1 Department of Computer Science, University of York, \\ YO10 5DD, UK \\ 2 Faculty of Engineering, Suez Canal university, Egypt \\ \{howaida, erh\}@cs.york.ac.uk
}

\begin{abstract}
This paper describes a new approach for embedding graphs on pseudo-Riemannian manifolds based on the wave kernel. The wave kernel is the solution of the wave equation on the edges of a graph. Under the embedding, each edge becomes a geodesic on the manifold. The eigensystem of the wave-kernel is determined by the eigenvalues and the eigenfunctions of the normalized adjacency matrix and can be used to solve the edge-based wave equation. By factorising the Gram-matrix for the wave-kernel, we determine the embedding co-ordinates for nodes under the wave-kernel. We investigate the utility of this new embedding as a means of gauging the similarity of graphs. We experiment on sets of graphs representing the proximity of image features in different views of different objects. By applying multidimensional scaling to the similarity matrix we demonstrate that the proposed graph representation is capable of clustering different views of the same object together.
\end{abstract}

Keywords: Wave Equation, Pseudo Riemannian manifolds, Edge-based Laplacian, Graph Embedding.

\section{Introduction}

Graph embeddings have found widespread use in machine learning and pattern recognition for the purposes of clustering, analyzing and visualizating relational data. However, they have also proved to be useful as a means of graph characterization. There are many examples in the literature including ISOmap [13], the Laplacian eigenmap [1, and the heat-kernel embedding [16, to name a few. Once embedded, a graph can be characterised using a feature-vector that characterises the point-set distribution resulting from the embedding [15]. This kind of representation is convenient since a Euclidean vector space makes available powerful geometric analysis tools for data analysis, not available for discrete or structural representations. However, such an embedding assumes that the original relational data is metric. Sometimes, however, this is not the case. This is the case when the matrix characterisation of the relational graph contains negative

\footnotetext{
* This work was supported by the EU FET project SIMBAD (213250). Edwin Hancock was supported by a Royal Society Wolfson Research Merit Award.
} 
eigenvalues, i.e. it is not positive semi-definite. Under these circumstances the graph embeds not into a Euclidean space, but into pseudo-Euclidean or Krein space 12. This problem has attracted relatively little attention in the literature. Our aim in this paper is to embed the nodes of a graph as points on the surface of a pseudo-Riemannian manifold in a pseudo-Euclidean space, and to use the resulting point-set as the basis from which to compute graph characteristics. To provide a framework for our study, we turn to the wave kernel. This is the solution of a wave equation, which is an important second-order linear partial differential equation that describes the propagation of a variety of waves. Crucially, the solutions are complex and therefore reside in a pseudo-Euclidean space. Although the wave equation has been extensively studied in the continuous domain, there has been relatively little effort devoted to understanding its behavior on a graph. In common with the heat kernel, the wave kernel can be defined in terms of a combinatorial Laplacian. However, in the case of the wave kernel this is the edge-based Laplacian, introduced by Friedman [6].

In this paper we explore how to solve the edge-based wave equation, in terms of the eigensystem of the edge-based Laplacian. Since the solution is a sinusoid, it contains both real and imaginary parts. Hence, we embed the nodes of the graph as points residing on a pseudo-Riemannian manifold, determined by the eigenvalues and eigenvectors of the edge-based Laplacian. In our experiments on graphs extracted from 2D image data, we use this matrix for the purpose of graph visualization. The remainder of this paper is organized as follows: In Section 2 we commence by embedding graphs onto pseudo Riemannian manifolds. First we show how to find the solution of the wave equation on a graph using the edge-based Laplacian in 92.1 . Then we construct the coordinate matrix for the pseudo-Euclidean embedding in $\$ 2.2$. Finally, \$2.3 is devoted to establishing the edge-based Laplacian matrix. In Section 3 we illustrate how to manipulate vectors in a pseudo Euclidean space, commencing by computing the square distance between any arbitrary pair of vectors in 3.1 In $\$ 3.2$ we show how to construct an orthonormal basis, and in 3.3 how to project vectors from a pseudo Euclidean space onto a 2D sub-space. Section 4 gives a brief review for the Hausdorff distance as a tool of comparing sets of unordered observations resulting from the embedding of the graphs. Section 5 presents our experimental evaluaton. Finally, conclusions are drawn and future directions of research suggested in Section 6

\section{Embedding Graphs into Pseudo Riemannian Manifolds}

\subsection{Edge-Based Wave Equation}

Friedman [6] has developed a graph-based version of the wave equation that has many of the properties of the classical Laplacian wave equation. The development is based on a variant of the combinatorial Laplacian referred to as the edgebased Laplacian. This graph theoretic version of the wave equation provides an interesting link with the continuous wave eqaution, and has a simple physical interpretation. The edges of the graph can be viewed as taut strings, joined 
together at the vertices. In fact, the edge-based Laplacian has been shown in the physics literature to be the "limiting case" of a "quantum wire" 8].

Graph theory defines a combinatorial Laplacian, $L$, as an operator on functions whose domain is the set of vertices of a graph. On the vertex-set the wave equation is $U_{t t}=-L U$ (the negative sign is due to that the combinatorial Laplacians are positive semi-definite). However, this wave equation fails to give a finite speed for wave propagation. As a result there is no simple link between the graph theoretic wave equation and its continuous counterpart. To overcome this problem a so-called edge-based wave equation $W_{t t}=-L_{E} W$ was introdiced by Friedman [6], where $L_{E}$ is the edge-based Laplacian, which is a better approxomation to the continuous Laplacian (i.e. the second derivative) than the combinatorial Laplacian $L$. The edge-based wave equation has unit wave propagation speed, while that based on the combinatorial Laplacian $L$ has infinite speed.

For the edge-based Laplacian, the eigenfunction $f$ satisfies $L_{E} f=\lambda f$ and $L f=0$ where $\Lambda_{E}=\{\lambda\}$ is the set of Laplacian eigenvalues. In fact, if $L$ is normalized and the graph under study has each edge weight equal to unity, then $L$ is similar to $\left(1-\cos \sqrt{L_{E}}\right)$. That is to say if $\Delta$ is a continuous Laplacian then $\widetilde{\Delta}=1-\cos \sqrt{-\Delta}$ is the corresponding combinatorial Laplacian. Hence, the eigenvalue $\lambda$ is in $\Lambda_{E}$ if and only if $(1-\cos \sqrt{\lambda})$ is in $\Lambda$ (the set of all eigenvalues of the combinatorial Laplacians). Note that $\Lambda_{E}$ is an infinite set of non-negative values (whose square roots are periodic), and exclude those which are multiples of $\pi$ from $\Lambda_{E}$. Recall that the general solution of the wave equation

$$
\begin{aligned}
W_{t t} & =-L_{E} W \\
\left.W\right|_{t=0} & =f \\
\left.W_{t}\right|_{t=0} & =g
\end{aligned}
$$

has the form

$$
W=\frac{\sin \left(\sqrt{L_{E}} t\right)}{\sqrt{L_{E}}} g+\cos \left(\sqrt{L_{E}} t\right) f
$$

For our work it suffices to compute the fundamental solution $W$ that satisfies $\left.W\right|_{t=0}=0$ and $\left.W_{t}\right|_{t=0}=1$, that is

$$
W=\frac{\sin \sqrt{L_{E}} t}{\sqrt{L_{E}}}
$$

Since, $L_{E}$ is positive semi-definite [6], $W$ can be approximated using the MacLaurin series, giving

$$
W=t\left[I-\frac{1}{6} L_{E} t^{2}\right]
$$

Now we can consider the nodes of the graph as residing on a pseudo-Riemannian manifold and the edges as geodesics on the manifold. 


\subsection{The Embedded Coordinates Matrix}

Positive definite Riemannian manifolds can be represented in one of two ways. Either a) their properties are defined intrinsically, or b) they can be regarded as subsets of a Euclidean space of higher dimension. Following the work of Nash [10/11 and Whitney [14, it has been known for some time that these approaches are equivalent, in the sense that any intrinsically defined Riemannian manifold can be embedded, with appropriate differentiability, into a Euclidean space. In 2, Clarke showed that the same situation holds in the case of pseudo-Riemannian manifolds, with metrics of indefinite signature.

The pseudo-Euclidean space generalizes the well-known Euclidean space to the case where inner products are indefinite. This effectively amounts to two Euclidean spaces, one of which has a positive semi-definite inner product and the second with a negative semi-definite inner product. For squared Euclidean distances, the embedding is determined by the pseudo Gram matrix $C=-\frac{1}{2} Q W Q$ derived from the kernel matrix $W$, where $Q=e e^{T}-I$ and $e=(1, \ldots, 1)^{T}$. If the matrix with the embedding co-ordinates as columns is $X^{T}$, then

$$
C=-\frac{1}{n} X X^{T}
$$

In the pseudo Euclidean space

$$
C=-\frac{1}{n} X\left(\begin{array}{cc}
M & 0_{(p+q) \times k} \\
0_{k \times(p+q)} & 0_{k \times k}
\end{array}\right) X^{T}
$$

where

$$
M=\left(\begin{array}{cc}
I_{p \times p} & 0_{p \times q} \\
0_{q \times p} & -I_{q \times q}
\end{array}\right)
$$

and $0_{k \times k}$ is the $k \times k$ matrix filled with zeros, and $p+q+k=n$. We can then write $X M X^{T}=\Phi \Lambda \Phi^{T}=\Phi|\Lambda|^{\frac{1}{2}} M|\Lambda|^{\frac{1}{2}} \Phi^{T}$, where $\Phi$ is the column-matrix of the eigenvectors and $\Lambda$ the diagonal matrix of the corresponding eigenvalues. The vectors are recovered via the transformation $X_{L}=\Phi_{L}\left|\Lambda_{L}\right|^{\frac{1}{2}}$, where $\Phi_{L}$ is the column-matrix of the selected eigenvectors and $\Lambda_{L}$ the diagonal matrix of the corresponding eigenvalues. Hence, the columns of $X_{L}$ are the vectors in the pseudo-Euclidean space.

\subsection{Edge-Based Eigenvalues and Eigenfunctions}

Before we experiment with our embedding, we need first to construct the edgebased Laplacian matrix. We follow the procedure given in [6] where the edgebased eigenvalues and eigenfunctions are determined using those of a normalized adjacency matrix. To commence, consider a finite graph denoted by $G=(V, E)$ with node-set $V$ and edge-set of edges $E \subseteq V \times V$, with all edges of unit weight. The elements of the adjacency matrix $A$ of the graph $G$ are

$$
A(u, v)=\left\{\begin{array}{lc}
1 & \text { if }(u, v) \in E \\
0 & \text { otherwise }
\end{array}\right.
$$


Let $T$ be a diagonal matrix whose elements are the degrees of the nodes of $G$, that is $T(u, u)=\sum_{v \in V} A(u, v)=\operatorname{deg}_{u}$. By dividing each row of the adjacency matrix $A$ by its corresponding $d e g_{u}$, we obtain the normalized adjacency matrix $\widetilde{A}$. For each eigenvalue, $\lambda$ of $\widetilde{A}$ there is a unique value of $\cos ^{-1}(\lambda) \in[0, \pi]$. The edge-based eigenvalues are $2 n \pi+\cos ^{-1}(\lambda)$ and $2(n+1) \pi-\cos ^{-1}(\lambda)$, where $\{n=0,1,2, \ldots\}$. Hence, if $\omega \in\{\Re \backslash n \pi\}$ then $\omega^{2}$ is an edge-based eigenvalue if and only if $\cos \omega$ is an eigenvalue of $\widetilde{A}$. For each corresponding eigenfunction, $f$, of $\widetilde{A}, f$ can be extended to obtain an edge-based eigenfunction [6]. To summarize, for the edge-based eigenpair $(f, \lambda)$, we have that:

$1-\cos \lambda$ is an eigenvalue of $\widetilde{A}$,

2- $f$ is an eigenfunction of $\widetilde{A}$; that is $\widetilde{A} f=\cos \lambda f$,

3- $L_{E} f=\lambda f$ and $L f=0$.

The existence of a complete set of eigenvalues and eigenfunctions for the continuous Laplacian has been demonstrated in 77. Friedman has extended the analysis to the edge-based Laplacian for finite graphs $[6$. To outline the theory, let $G$ be a finite graph. For $G$ there exists eigenpairs $f_{i}, \lambda_{i}$ for the edge-based Lalacian, such that

$1-0 \leqq \lambda_{1} \leqq \lambda_{2} \leqq \ldots$

2- $f_{i}$ satisfies the Dirichlet (Neumann) conditions,

3- $f_{i}$ forms a complete orthonormal basis for $L_{D i r}^{2}(G)\left(L^{2}(G)\right)$,

$4-\lambda_{i} \rightarrow \infty$.

Physically, the equations $L_{E} f=\lambda f$ and $L f=0$ describe the vibrational modes associated with a taut strings on each edge that are joined together at the vertices. If we excite or "pluck" the system, it would produce tones with frequencies $\sqrt{\lambda}$, with $\lambda$ ranging over the edge-based eigenvalues.

\section{Pseudo Euclidean Space}

A pseudo Euclidean space is an n-dimensional space $r_{1}, r_{2}, \ldots, r_{n}$ where $r_{i}=r$ or $i r$ and $r$ is a set of real numbers and $i=\sqrt{-1}$. In this section we describe how to manipulate vectors in a pseudo Euclidean space. Firstly, we explain how to compute the square distance between any arbitrary pair of vectors. Secondly, we show how to construct an orthonormal basis. Thirdly, we show how to project vectors from a pseudo Euclidean space onto a 2D sub-space.

\subsection{Distance Function}

With a pseudo Euclidean space $R^{n}$ we assign a symmetric bilinear form $\rho$ : $R^{n} \times R^{n} \rightarrow R, \rho(x, y)=x^{T} S y$ where $S$ is the matrix whose elements $s_{i j}=$ $\frac{1}{2}\left(d_{i i}^{2}+d_{j j}^{2}-d_{i j}^{2}\right) ; d$ is a distance function with pairwise distances $d_{i j}$ for all $1 \leqslant i, j \leqslant n$. For any two vectors $x, y \in R^{n}, \rho(x, y)$ is the inner product of $x$ and $y$ and $\|x-y\|^{2}=\rho(x-y, x-y)$ is the squared distance between $x$ and $y$. Since $S$ is real symmetric, there is an orthogonal matrix $\Psi$ and a diagonal matrix $\Gamma$ such that $S=\Psi \Gamma \Psi^{T}$, the elements $\delta_{i}$ of $\Gamma$ are the eigenvalues of $S$ arranged in order and the columns of $\Psi$ are the correspondingly ordered eigenvectors. It is 
worth mentionng that if the matrix $S$ has negative eigenvalues, then the squared distance between two vectors in the pseudo Euclidean space may be negative. It for this reason that we do not speak about "distance" between vectors in pseudo Euclidean space. Moreover, the fact that the squared distance between two vectors vanishes does not imply that these two vectors are the same. This is not the case in a Euclidean space.

\subsection{An Orthonormal Basis}

The columns $\left\{b_{i}\right\}, i=1, \ldots, n$ of the matrix $B=I \Psi$ represent an orthogonal basis of $R^{n}$, since $S$ is the matrix of $\rho$ with respect to the natural basis $\left\{e_{i}\right\}, i=1, \ldots, n$ where $e_{i}=\left(0, \ldots, 1_{i}, \ldots, 0\right)$. We can therefore write the bilinear form $\rho$ with respect to the basis $b_{i}$ as $S_{b}=\Psi^{T} S \Psi$, so that $S_{b}=\Gamma$. For any two vectors $x$ and $y$ in $R^{n}, \rho(x, y)=x^{T} S y=\left[x_{b}^{T} \Psi^{T}\right]\left[\Psi S_{b} \Psi^{T}\right]\left[\Psi y_{b} \Psi^{T}\right]$. Hence, $\rho(x, y)=x_{b}^{T} S_{b} y_{b}=$ $x_{b}^{T} \Gamma y_{b}$. Accordingly, the inner product of $x$ and $y$ can be written as $\rho(x, y)=$ $\sum_{i=1}^{n} \delta_{i}\left(x_{b}\right)_{i}\left(y_{b}\right)_{i}$ and the squared distance as $\|x-y\|^{2}=\sum_{i=1}^{n} \delta_{i}\left(\left[x_{b}\right]_{i}-\left[y_{b}\right]_{i}\right)^{2}$. The matrix $X_{b}=X \Psi$ has as columns the coordinates with respect to the basis $\left\{b_{i}\right\}$. Conversely, the coordinate matrix $X_{e}=X_{o} \Psi^{T}$ has as columns the coordinate vectors with respect to an orthogonal natural basis.

Let us define a diagonal matrix $J=\operatorname{diag}\left(j_{i j}\right)$ with elements

$$
j_{i}= \begin{cases}1 & \delta_{i}>0 \\ 0 & \delta_{i}=0 \\ -1 & \delta_{i}<0\end{cases}
$$

and $i=1, \ldots, n$, Furthermore, let $\widetilde{\Gamma}=\operatorname{diag}\left(\gamma_{i}\right)$ where

$$
\gamma_{i}= \begin{cases}\left|\delta_{i}\right| & \text { if } \delta_{i} \neq 0 \\ 1 & \text { otherwise }\end{cases}
$$

Now consider the matrix $\widetilde{\Psi}=\Psi \widetilde{\Gamma}^{-\frac{1}{2}}$. The first $l$ columns of this matrix are orthonormal vectors with respect to $\left\{b_{i}\right\}$. To show this consider the matrix

$$
\widetilde{\Psi}^{T} S \widetilde{\Psi}=\widetilde{\Gamma}^{-\frac{1}{2}} \Psi^{T}\left[\Psi S_{b} \Psi^{T}\right] \Psi \widetilde{\Gamma}^{-\frac{1}{2}}=\widetilde{\Gamma}^{-\frac{1}{2}} \Gamma \widetilde{\Gamma}^{-\frac{1}{2}}=J
$$

The diagonal elements $J_{i}, i=1, \ldots, l$ are either 1 or -1 , while the remainder are zeros. Hence, the first $l$ columns of the matrix $\widetilde{B}=B \widetilde{\Gamma}^{-\frac{1}{2}}$ form an orthonormal basis of $R^{l}$. Finally, for the matrix $X_{e}$ whose columns are the co-ordinate vectors in the pseudo Euclidean space with respect to the natural basis $\left\{e_{i}\right\}_{i=1, \ldots, n}$, the corresponding matrix of coordinates with respect to the orthonormal basis $\left\{b_{i}\right\}_{i=1, \ldots, n}$ is $X_{\widetilde{b}}=X_{e} \widetilde{\Psi}$.

\subsection{Projection into a 2D Subspace}

Suppose we order eigenvalues of the matrix $S$ so that first $l^{+}$eigenvalues are positive, the following $l^{-}$are negative and the remainder are zero, where $l=$ $l^{+}+l^{-}$. As a result $\left\{b_{i}\right\}_{1 \leq i \leq l}$, and the first $l$ columns of the matrix $B$ given in 
Section 3.2 form an orthogonal basis of the space $R^{l}$. Using the first $l$ columns of the matrix $\widetilde{\Psi}$, we can locate the projections of the column vectors of the matrix $X$ onto the space $R^{l}$ with respect to $\left\{b_{i}\right\}_{1 \leq i \leq l}$ as $X_{l}=B \widetilde{\Psi}^{T}$. To obtain the coordinates of $X_{l}$ with respect to the orthonormal basis $\widetilde{b}_{i}$, we construct the matrix $X_{l_{\tilde{b}}}=\widetilde{B} \widetilde{D}_{l}^{-\frac{1}{2}} \Psi_{l}^{T}=\left(\bar{p}_{1}\left|\bar{p}_{2}\right| \ldots\right)$, where $\widetilde{D}_{l}=\operatorname{diag}\left(\gamma_{i}\right), 1 \leq i \leq l$ is the $l^{\text {th }}$ leading principle submatrix of $\widetilde{D}$ and $\bar{p}_{i}$ is the projected coordinate vector of the $i^{\text {th }}$ node of $G$. Again we can define the inner product of two arbitrary vectors, $x$ and $y$, as $\rho(x, y)=\sum_{i=1}^{n} \delta_{i}\left(x_{b}\right)_{i}\left(y_{b}\right)_{i}$ and the squared distance as $\|x-y\|^{2}=\sum_{i=1}^{n} \delta_{i}\left(\left[x_{b}\right]_{i}-\left[y_{b}\right]_{i}\right)^{2}$.

To avoid problems associated with dealing with a space of high dimensionality, we will ignore the dimensions for which the eigenvalues are small in magnitude. Therefore, if we arrange the eigenvalues in descending order by their absolute values, the first $k$ eigenvalues (typically $k=2$ or 3 ) where $k<l$ will span a space $R^{k}$ in which we can project the exact vector representation of the pseudo Euclidean space $R^{n}$.

\section{Hausdorff Distance}

We experiment with the wave kernel embedding as a graph characterization for the purposes of graph-matching. We represent the graphs under study using sets of coordinate vectors corresponding to the embedded node position, and compute the similarity of the sets resulting from different graphs using the robust modified Hausdorff distance. The Hausdorff distance provides a means of computing the distance between sets of unordered observations when the correspondences between the individual items are unknown. In its most general setting, the Hausdorff distance is defined between compact sets in a metric space. Given two such sets, we consider for each point in one set is the closest point in the second set. The modified Hausdorff distance is the average over all these values. More formally, the modified Hausdorff distance $(M H D)$ [4] between two finite point sets $A=\left\{\bar{p}_{1}^{A}\left|\bar{p}_{2}^{A}\right| \ldots \mid \bar{p}_{\left|V_{A}\right|}^{A}\right\}$ and $B=\left\{\bar{p}_{1}^{B}\left|\bar{p}_{2}^{B}\right| \ldots \mid \bar{p}_{\left|V_{B}\right|}^{B}\right\}$ representing the projected embeddings of the graphs $G_{A}\left(V_{A}, E_{A}\right)$ and $G_{B}\left(V_{B}, E_{B}\right)$

$$
H(A, B)=\max (h(A, B), h(B, A))
$$

where the directed modified Hausdorff distance from A to B is defined to be

$$
h(A, B)=\frac{1}{N_{A}} \sum_{a \in A} \min _{b \in B}\|a-b\|
$$

and $\|$.$\| is some underlying norm on the points of A and B (e.g., the L2 or$ Euclidean norm). We can now write the distances between two graphs as follows:

$$
\left.h_{M H D}\left(G_{A}, G_{B}\right)=\frac{1}{\left|V_{A}\right|} \sum_{i \in V_{A}} \min _{j \in V_{B}}\left\|\bar{p}_{i}^{A}-\bar{p}_{j}^{B}\right\|\right)
$$




\section{Experiments and Results}

In our experiments we aim to investigate weather the edge-based wave kernel embedding can be used as a graph characterization, for gauging the similarity of graphs, and hence clustering them. We use the standard CMU,MOVI and chalet house sequences as our data set [9. These data sets contain different views of model houses from equally spaced viewing directions. We have used ten views for each of the three houses. We have also applied our method to objects selected from the COIL database. This contains 72 different views of each object from equally spaced viewing directions. For each image corner features are extracted, and Delaunay graphs representing the arrangement of the feature points are constructed.

To commence, we compute the eigensystem of the edge-based Laplacian from the eigensystem of the normalized adjacency matrix, and hence compute the edge-based Laplacian matrix introduced in Section 2.3. The edge-based wave kernel then is computed as described in Section 2.1 with the values of $t=$ 10.0, 1.0,0.1 and 0.01. From the wave-kernel we compute the embedding coordinate matrix, whose columns are the coordinates of the embedded nodes in a pseudo-Euclidean space. Finally, we project the co-ordinate vectors onto a pseudo-Euclidean space with low dimension using the orthonormal basis as shown in Section 3. With the vector representations residing in a low dimension space we construct the distances matrices between the thirty different graphs using the modified Hausdorff distance [5]. Finally, we subject the distance matrices

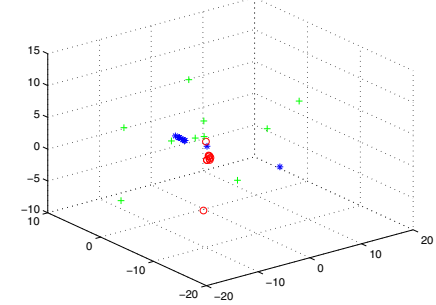

(a) $\mathrm{t}=10.0$

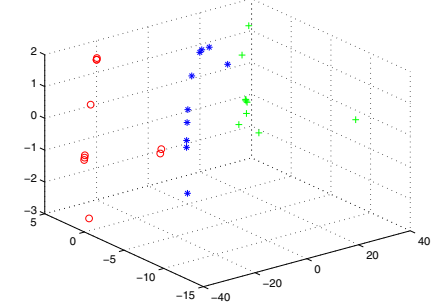

(c) $\mathrm{t}=0.1$

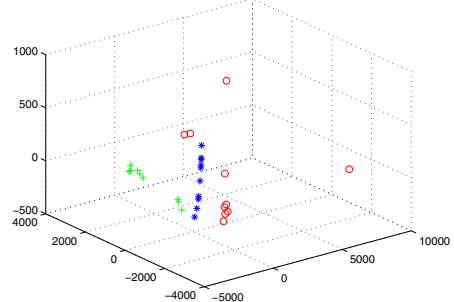

(b) $\mathrm{t}=1.0$

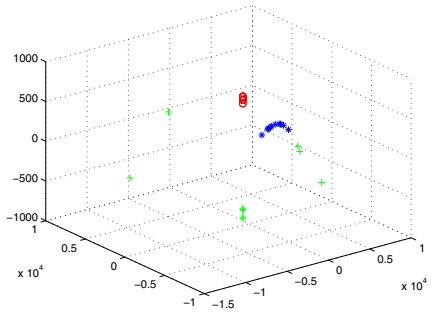

(d) $\mathrm{t}=0.01$

Fig. 1. MDS embedding obtained when using the Wave Kernel for the houses data 


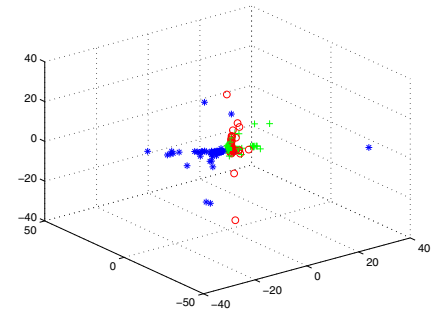

(a) $\mathrm{t}=10.0$

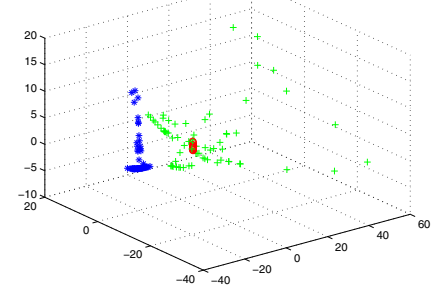

(c) $\mathrm{t}=0.1$

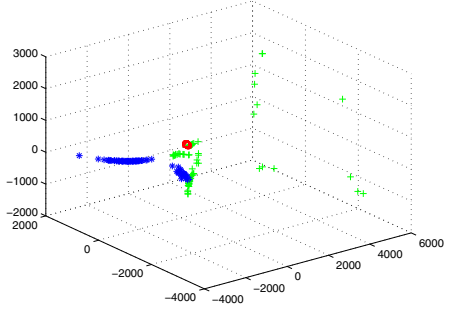

(b) $\mathrm{t}=1.0$

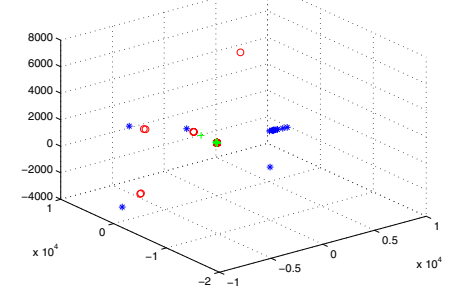

(d) $\mathrm{t}=0.01$

Fig. 2. MDS embedding obtained when using the Wave Kernel for the COIL data

Table 1. A rand index vs. $t$

\begin{tabular}{|c|c|c|c|c|}
\hline & $\mathrm{t}=10$ & $\mathrm{t}=1.0$ & $\mathrm{t}=0.1$ & $\mathrm{t}=0.01$ \\
\hline Houses data & 0.2333 & 0.0000 & 0.0333 & 0.1000 \\
\hline COIL data & 0.3333 & 0.3333 & 0.3333 & 0.7000 \\
\hline
\end{tabular}

to multidimensional scaling $M D S[3$ to embed them into a $2 \mathrm{D}$ space. Here each graph is represented by a single point. Figure 1 shows the results obtained using the modified Hausdorff distance. The subfigures are ordered from left to right (up to down), using $t=10.0,1.0,0.1$ and 0.01 in the wave kernel. We have also investigated the COIL data, and the results are shown in Figure 2 ,

To investigate the data in more detail Table 1 shows the Rand index for the data as a function of $t$. This index is computed as follows: 1) compute the mean for each cluster; 2) compute the distance from each point to each mean; 3) if the distance from correct mean is smaller than those to remaining means, then classification is correct, if not then classification is incorrect; 4) compute the Rand-index (incorrect/(incorrect+correct)).

There are number of conclusions to be drawn from the plots. First, the wave kernel gives good separation of the objects into distinct clusters particularly for values of $t$ close to 1 . Second, the individual objects form clear trajectories in the embedding space which correlate will with the view ordering. 


\section{Conclusion and Future Plan}

In this paper we have established a procedure to embed the nodes of a graph into a pseudo-Riemannian manifold using the wave kernel, which is the solution of an edge-based wave equation. The edge-based Laplacian matrix was constructed using the eigensystem of the normalized adjacency matrix. Based on experiments on objects from two datasets (the York Model House and COIL datasets), we are confident that an edge-based wave kernel embedding can be used for the purpose of graph characterization.

To take this work further if the nodes of a graph residing on a pseudo-Riemannian manifold, then we can associate curvatures with the edges of the graph since these can be viewed as geodesics on the manifold [15. In future research aimed at developing the work reported in this paper, we aim to investigate if we can use the geometry of the pseudo-Riemannian manifold to characterize graphs.

\section{References}

1. Belkin, M., Niyogi, P.: Laplacian eigenmaps and spectral techniques for embedding and clustering. In: Leen, T.K., Dietterich, T.G., Tresp, V. (eds.) Advances in neural information processing systems, vol. 14. MIT Press, Cambridge (2002)

2. Clarke, C.J.S.: On the global isometric embedding of pseudo-riemannian manifolds. In: Proceedings of Royal Society of London. A, vol. 314, pp. 417-428 (1970)

3. Cox, T., Cox, M.: Multidimensional Scaling. Chapman-Hall, Boca Raton (1994)

4. Dubuisson, M., Jain, A.: A modified hausdorff distance for object matching, pp. 566-568 (1994)

5. ElGhawalby, H., Hancock, E.R.: Measuring graph similarity using spectral geometry. In: Campilho, A., Kamel, M.S. (eds.) ICIAR 2008. LNCS, vol. 5112, pp. 517-526. Springer, Heidelberg (2008)

6. Friedman, J., Tillich, J.P.: Wave equations for graphs and the edge based laplacian. Pacific Journal of Mathematics 216(2), 229-266 (2004)

7. Gilbarg, D., Trudinger, N.S.: Elliptic Partial Differential Equations of Second Order. Springer, Heidelberg (1983)

8. Hurt, N.E.: Mathematical physics of quantum wires and devices. Kluwer Academic Publishers, Dordrecht (2000)

9. Luo, B., Wilson, R.C., Hancock, E.R.: Spectral embedding of graphs. Pattern Recogintion [36], 2213-2230 (2003)

10. Nash, J.F.: C1-isometric imbeddings. Ann. Math. 60, 383-396 (1954)

11. Nash, J.F.: The imbedding problem for riemannian manifolds. Ann. Math. 63, 20-63 (1956)

12. Pekalska, E., Haasdonk, B.: Kernel discriminant analysis for positive definite and indefinite kernels. IEEE transactions on pattern analysis and machine intelligence 31(6), 1017-1032 (2009)

13. Tenenbaum, J.B., de Silva, V., Langford, J.C.: A global geometric framework for nonlinear dimensionality reduction. Science 290, 2319-2323 (2000)

14. Whitney, H.: Differentiable manifolds. Ann. of Math. 37(2), 645-680 (1936)

15. Xiao, B., Hancock, E.R., Wilson, R.C.: Graph characteristics from the heat kernel trace. Pattern Recognition 42(11), 2589-2606 (2009)

16. Xiao, B., Hancock, E.R., Yu, H.: Manifold embeddingforshapeanalysis. Neurocomputing 73, 1606-1613 (2010) 